Ann. Sci. forest., 1980, 37 (1), 1-18.

\title{
Seuils de signification des coefficients de corrélation génotypique, phénotypique et environnementale. Etude du cas d'un test clonal
}

\author{
G. NEPVEU \\ Station de Recherches sur la Qualité des Bois \\ Centre national de Recherches forestières, I.N.R.A., \\ Champenoux, 54280 Seichamps
}

\section{Résumé}

Cet article est une application très simple de recherches développées par Tallis et Scheinberg. Il rappelle l'expression de la variance d'échantillonnage de l'estimation de coefficients de corrélation (génétique, génotypique, due à l'environnement, phénotypique) obtenus en analyse de variancecovariance.

II particularise au cas d'un test clonal et montre que l'on commet une erreur parfois importante en jugeant la signification des corrélations génotypique et phénotypique uniquement à l'aide des caractéristiques du dispositif : nombre de clones, nombre d'individus par clone.

\section{Introduction}

Cet article ne constitue qu'une application très simple de recherches développées par Tallis (1959) et Scheinberg (1966).

Son but est de mettre en évidence le danger de certaines approximations assez couramment acceptées en France notamment dans des tests concernant la signification des corrélations génotypique et phénotypique.

Il est vrai que le calcul des coefficients de corrélation génotypique et phénotypique sert surtout aujourd'hui à fournir une matrice pour l'établissement d'index de sélection faisant intervenir un nombre parfois élevé de caractères; on pourrait dans ce cadre discuter de l'importance réelle de la signification des coefficients de corrélation.

II reste cependani des cas où l'on sélectionne sur un ou deux caractères et où l'on désire connaître les conséquences de l'opération sur d'autres paramètres ; il est alors important de lester avec une certaine rigueur les liaisons entre caractères.

Dans un premier chapitre, nous rappellerons quelques résultats permettant, dans un cas très général, d'estimer la variance d'échantillonnage de l'estimation de coefficients de corrélation obtenus en analyse de variance-covariance ; nous en donnerons un intervalle de confiance sous l'hypothèse de nullité.

En second lieu, nous particulariserons au cas d'un test clonal, donnerons les expressions des variances d'échantillonnage des coefficients de corrélations génoty- 
pique, phénotypique ef due au milieu, ef vérifierons si les tests de signification adoptés de façon courante pour ces coefficients sont satisfaisants.

Dans un troisième chapitre, nous étudierons un exemple.

\section{1. - Variances d'échantillonnage de l'estimation de coefficients de corrélation obtenus en analyse de variance-covariance}

Nous nous placerons dans le cas le plus simple qui puisse se poser au généticien : celui d'une analyse de variance-covariance à un facteur, les effets étudiés pouvant être des effets "clone », «famille de demi-frères", ou même «famille de pleinfrères " que nous désignerons sous le vocable de « groupe ".

Nous choisirons un test équilibré ef appellerons $n$ le nombre d'individus par groupe et $p$ le nombre de groupes.

Nous attribuerons les indices 1 et 2 aux deux caractères que l'on désire corréler.

\section{1. - Modèle}

Les individus étudiés sont considérés comme un échantillon au hasard d'une population ; ils permettent d'estimer des paramètres de la population pour les caractères étudiés.

Pour chaque caractère, la valeur observable d'un individu $y_{i j}$ est une variable aléatoire qu'on écrit :

$$
y_{i j}=\mu+g_{i}+e_{i j}
$$

avec $\mu$ : moyenne générale du caractère dans la population de référence

$g_{i}$ : variable aléatoire représentant l'effel du groupe $i(i=1, \ldots, p)$

$\mathbf{e}_{\mathrm{ij}}$ : variable aléatoire représentant la déviation intragroupe $(j=1, \ldots, n)$.

Remarque : On insistera auprès du lecteur sur le fait que pour considérer $g_{i}$ comme une variable aléatoire, il faut que les groupes forment un échantillon au hasard de la population de référence.

\section{2. - Tableau d'analyse de variance-covariance}

\section{TABLEAU 1}

Analysis of variance and covariance

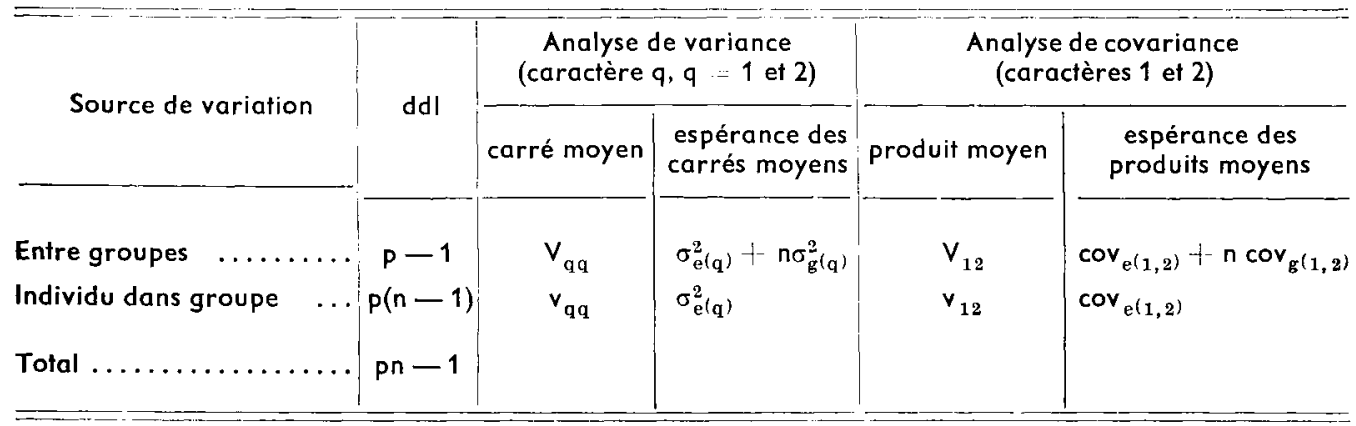


avec $\sigma_{\mathrm{g}(\mathrm{q})}^{2} \quad$ : variance entre groupes dans la population du caractère $q(q=1$ et 2$)$

$\sigma_{\mathrm{e}(q)}^{2} \quad$ : variance intragroupe dans la population du caractère $q$

$\operatorname{cov}_{\mathrm{g}(1,2)}$ : covariance entre groupes dans la population des caractères 1 et 2 .

$\operatorname{cov}_{e}(1,2)$ : covariance intragroupe dans la population des caractères 1 et 2 .

\section{3. - Rappel sur les moments des carrés moyens}

Les carrés ef coproduits moyens $\mathrm{V}_{\mathrm{qq}}, \mathrm{V}_{12}, \mathrm{v}_{\mathrm{qq}}, \mathrm{v}_{12}$ sont des variables aléatoires dont les espérances figurent dans le tableau d'analyse de variance-covariance.

Les résultats de Fisher (1928) permettent de définir les variances (covariances) de (entre) ces variables aléatoires à partir de leurs espérances.

Elles s'écrivent de façon générale :

$$
\begin{gathered}
\operatorname{var}\left(V_{q r}\right)=\frac{E\left(V_{q q}\right) \times E\left(V_{r r}\right)+\left[E\left(V_{q r}\right)\right]^{2}}{d d l \text { affectés à } V_{q r}} \\
\operatorname{cov}\left(V_{q r}, V_{s t}\right)=\frac{E\left(V_{q s}\right) \times E\left(V_{r t}\right)+E\left(V_{q t}\right) \times E\left(V_{r s}\right)}{\text { ddl affectés à } V_{q r}}
\end{gathered}
$$

On peut donner une estimation de ces variances ef covariances (Tallis, 1959, op. cit.) :

$$
\begin{aligned}
& \widehat{\operatorname{var}\left(V_{q r}\right)}=\frac{V_{q q} \times V_{r r}+\left(V_{q r}\right)^{2}}{d d l \text { affectés à } V_{q r}} \\
& \widehat{\operatorname{cov}\left(V_{q r}, V_{s t}\right)}=\frac{V_{q s} \times V_{r t}+V_{q t} \times V_{r s}}{d d l \text { affectés à } V_{q r}}
\end{aligned}
$$

1.4. - Interprétation génétique des termes $\sigma_{\mathrm{g}(\mathrm{q})}^{2}, \sigma_{\mathrm{e}(\mathrm{q})}^{2}$ (respectivement $\operatorname{cov}_{(1,2)}$ et $\operatorname{cov}_{\mathrm{e}}(1,2)$ ) dans différents cas

Nous nous contenterons de quelques rappels sans revenir sur les conditions nécessaires à ces interprétations (panmixie...).

Nous appellerons :

$\sigma_{\mathrm{A}(q)}^{2}$ variance des effets génétiques additifs

$\sigma_{\mathrm{D}(q)}^{2}$ variance des effets génétiques non additifs

$\sigma_{\mathrm{G}(q)}^{2}$ variance génotypique $\left(=\sigma_{\mathrm{A}(q)}^{2}+\sigma_{\mathrm{D}(q)}^{2}\right)$

$\sigma_{\mathrm{E}(q)}^{2}$ variance des effets dus à l'environnement

$\sigma_{\mathrm{P}(q)}^{2}$ variance phénotypique $\left(=\sigma_{\mathrm{A}(\mathrm{q})}^{2}+\sigma_{\mathrm{D}(\mathrm{q})}^{2}+\sigma_{\mathrm{E}(\mathrm{q})}^{2}\right)$.

\begin{tabular}{|c|c|c|c|}
\hline & Clones & Familles de demi-frères & $\begin{array}{l}\text { Familles de plein frères } \\
\text { non apparentées }\end{array}$ \\
\hline$\sigma_{\mathbf{g}(\mathbf{q})}^{2}$ & $\sigma_{G(q)}^{2}$ & $\frac{1}{4} \sigma_{A(q)}^{2}$ & $\frac{1}{2} \sigma_{A^{(q)}}^{2}+\frac{1}{4} \sigma_{D(q)}^{2}$ \\
\hline$\sigma_{\mathbf{e}(q)}^{2}$ & $\sigma_{\mathrm{E}(q)}^{2}$ & $\frac{3}{4} \sigma_{A(q)}^{2}+\sigma_{D(q)}^{2}+\sigma_{E(q)}^{2}$ & $\frac{1}{2} \sigma_{A(q)}^{2}+\frac{3}{4} \sigma_{D(q)}^{\delta}+\sigma_{E(q)}^{2}$ \\
\hline$\sigma_{\mathbf{g}(\mathbf{q})}^{2}+\sigma_{\mathbf{e}(\mathbf{q})}^{2}$ & $\sigma_{\mathbf{P}(\mathbf{q})}^{2}$ & $\sigma_{\mathbf{P}(\mathbf{q})}^{2}$ & $\sigma_{\mathbf{P}(q)}^{2}$ \\
\hline
\end{tabular}

\section{TABLEAU 2}

Interprétation génétique des fermes $\sigma_{\mathrm{g}(\mathrm{q})}^{2}$, $\sigma_{\mathrm{e}(\mathrm{q})}^{2}$ (respectivement $\operatorname{cov}_{\mathrm{g}(1,2)}$ et $\operatorname{cov}_{\mathrm{e}(1,2)}$ ) dans différents cas Genetic interpretation of $\sigma_{\mathrm{g}(\mathrm{q})}^{2}, \sigma_{\mathrm{e}(\mathrm{q})}^{2}\left(\operatorname{cov}_{\mathrm{g}(1,2)}\right.$ and $\operatorname{cov}_{\mathrm{e}(1,2)}$ respectively) in some cases 
On voit qu'il est possible d'obtenir des estimations de $\sigma_{\mathrm{A}(q)}^{2}, \sigma_{\mathrm{G}(q)}^{2}, \sigma_{\mathrm{E}(q)}^{2}$ ou $\sigma_{\mathbf{P}(q)}^{2}$ (respectivement $\left.\operatorname{cov}_{\mathrm{A}(1,2)}, \operatorname{cov}_{\mathrm{G}(1,2)}, \operatorname{cov}_{\mathrm{E}(1,2)}, \operatorname{cov}_{\mathrm{P}(1,2)}\right)$ à l'aide d'une combinaison linéaire de $V_{q q}$ ef $V_{q q}$ (respectivement $V_{12}$ ef $v_{12}$ ) de la forme $\lambda V_{q a}+u v_{q a}$ (respectivement $\lambda V_{12}$ ef $\mu v_{12}$ ).

Nous désignerons par le terme général $\theta_{\mathrm{qq}}$ (respectivement $\theta_{12}$ ) les termes $\sigma_{\mathrm{A}(q)}^{2}, \sigma_{\mathrm{G}(q)}^{2}, \sigma_{\mathrm{E}(q)}^{2}, \sigma_{\mathrm{P}(q)}^{2},\left(\right.$ respectivement $\left.\operatorname{cov}_{\mathrm{A}(1,2)}, \operatorname{cov}_{\mathrm{G}(1,2)}, \operatorname{cov}_{\mathrm{E}(1,2)}, \operatorname{cov}_{\mathrm{P}(1,2)}\right)$.

On a $\hat{\theta}_{\mathrm{qq}}=\lambda V_{\mathrm{qq}}+\mu v_{\mathrm{qq}}$ qui est une variable aléatoire d'espérance $\theta_{\mathrm{qq}}$ et de variance :

$$
\lambda^{2} \operatorname{var} \mathrm{V}_{\mathrm{qq}}+\mu^{2} \operatorname{var} \mathrm{v}_{\mathrm{qq}}+2 \lambda \mu \operatorname{cov}\left(\mathrm{V}_{\mathrm{qq}}, \mathrm{v}_{\mathrm{qq}}\right) \text {. }
$$

Or $V_{q}$ et $v_{q}$ sont supposés indépendants par le modèle d'analyse de variance, d'où :

$$
\operatorname{var} \hat{\theta}_{\mathrm{qq}}=\lambda^{2} \operatorname{var} \mathrm{V}_{\mathrm{qq}}+\mu^{2} \operatorname{var} \mathrm{v}_{\mathrm{qq}} \text {. }
$$

De même, l'estimation de la covariance $\hat{\theta}_{12}=\lambda V_{12}+\mu v_{12}$ a pour espérance $\theta_{12}$ et pour variance $\lambda^{2}$ var $V_{12}+\mu^{2}$ var $V_{12}$.

On montrerait de même que

$$
\begin{aligned}
& \operatorname{cov}\left(\hat{\theta}_{\mathrm{qq}}, \hat{\theta}_{12}\right)=\lambda^{2} \operatorname{cov}\left(\mathrm{V}_{\mathrm{qq}}, \mathrm{V}_{12}\right)+\mu^{2} \operatorname{cov}\left(\mathrm{v}_{\mathrm{qq}}, \mathrm{v}_{12}\right) \\
& \operatorname{cov}\left(\hat{\theta}_{11}, \hat{\theta}_{22}\right)=\lambda^{2} \operatorname{cov}\left(\mathrm{V}_{11}, \mathrm{~V}_{22}\right)+\mu^{2} \operatorname{cov}\left(\mathrm{v}_{11}, \mathrm{v}_{22}\right) .
\end{aligned}
$$

1.5. - Valeurs prises par $\lambda$ et $\mu$ dans différents cas ; rappels sur l'interprétation des $\theta_{\mathrm{qq}}$ obtenus (respectivement $\theta_{12}$ ).

TABLEAU 3

Voleurs prises par $\lambda$ et $\mu$ dans différents cas; rappe/s sur l'interprétation des $\theta_{\mathrm{qq}}$ obtenus (respectivement $\theta_{12}$ ) Values of $\lambda$ and $\mu$ in some cases ; interpretation of $\theta_{\mathrm{q} 19}$ calculated $\left(\theta_{12}\right.$ respectively)

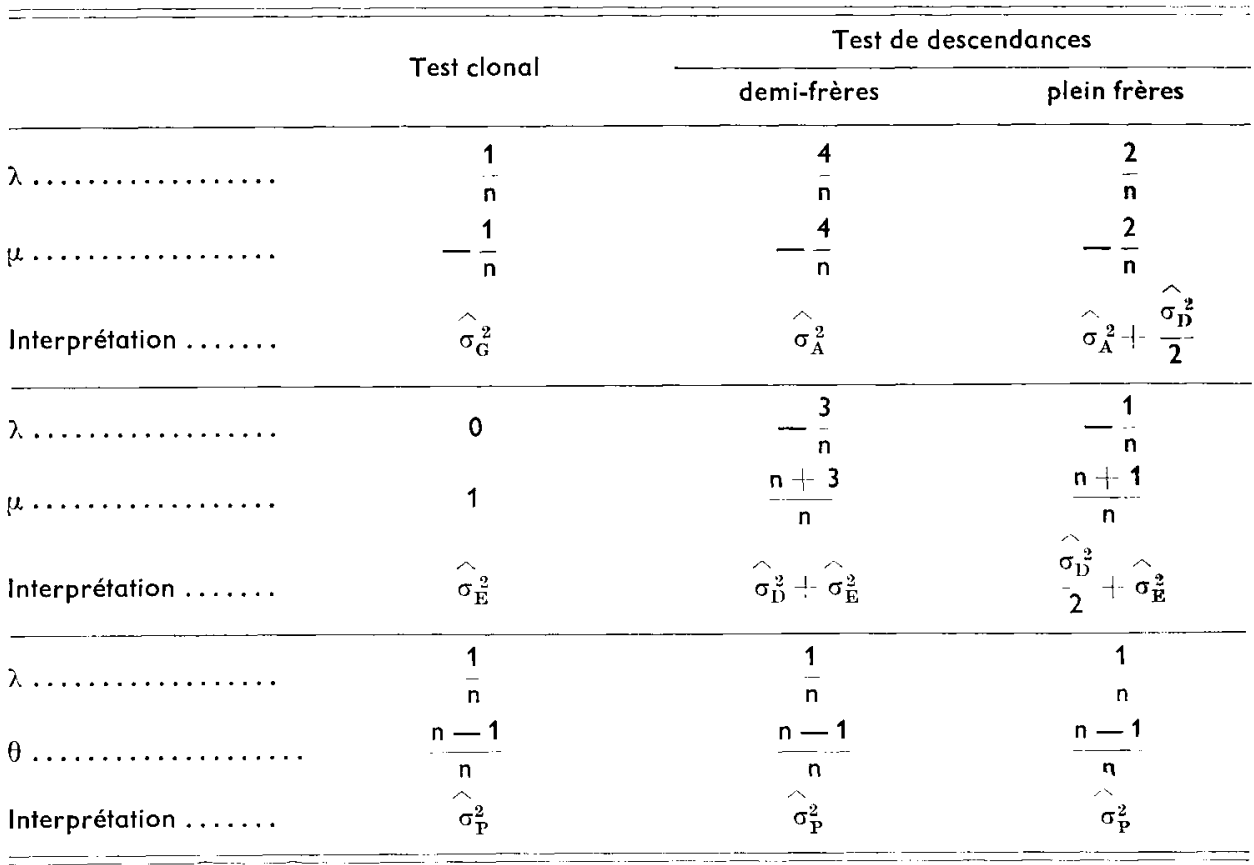




\section{6. - Définition des coefficients de corrélation}

De même qu'on définit des variances (et covariances) génétique, génotypique, due à l'environnement, phénotypique, on peut définir entre deux caractères 1 et 2 , des coefficients de corrélation génétique, génotypique, due à l'environnement, phénotypique de la forme

$$
r_{\theta(1,2)}=\frac{\theta_{12}}{\sqrt{\theta_{11} \times 0_{22}}}
$$

On va s'attacher en 1.7 à définir la variance d'échantillonnage de l'estimation de $r_{\theta(1,2)}$ que l'on peut obtenir par l'analyse de variance-covariance.

\section{7. - Calcul de var $\left(\mathrm{r}_{\theta(1}, 1\right)$}

On a :

$$
r_{\theta(1,2)}=\frac{\hat{\theta}_{12}}{\sqrt{\hat{\theta}_{11} \times \hat{\theta}_{22}}}
$$

$\hat{r}_{\theta(1,2)}$ est une fonction qui dépend de trois variables aléatoires $\hat{\theta}_{12}, \hat{\theta}_{11}$ et $\hat{\theta}_{22}$ qui ont respectivement pour espérances $\theta_{12}, \theta_{11}$ et $\theta_{22}$.

Kendall et Stuart (1963, p. 232) donnent une expression générale approchée de la variance d'une fonction $g$ qui dépend de variables aléatoires $x_{1}, x_{2}, \ldots, x_{k}$ de moyennes $m_{1}, m_{2}, \ldots, m_{k}$ :

$$
\operatorname{var} g=\sum_{i, j=1}^{k} \frac{\partial g}{\partial m_{i}} \times \frac{\partial g}{\partial m_{j}} \operatorname{cov}\left(x_{i}, x_{j}\right) \text {. }
$$

Appliqué au cas qui nous intéresse :

$$
\begin{aligned}
& \operatorname{var} \hat{r_{\theta}}=\frac{2 \partial r_{\theta}}{\partial \theta_{12}} \times \frac{\partial r_{0}}{\partial \theta_{11}} \times \operatorname{cov}\left(\hat{\theta}_{12}, \hat{\theta}_{11}\right)+\frac{2 \partial r_{0}}{\partial \theta_{12}} \times \frac{\partial r_{\theta}}{\partial \theta_{22}} \times \operatorname{cov}\left(\hat{\theta}_{12}, \hat{\theta}_{22}\right) \\
& +\frac{2 \partial r_{0}}{\partial \theta_{11}} \times \frac{\partial r_{0}}{\partial \theta_{22}} \times \operatorname{cov}\left(\hat{\theta}_{11}, \hat{\theta}_{22}\right)+\left(\frac{\partial r_{0}}{\partial \theta_{12}}\right)^{2} \times \operatorname{var}\left(\hat{\theta}_{12}\right)+\left(\frac{\partial r_{0}}{\partial \theta_{11}}\right)^{2} \times \operatorname{var}\left(\hat{\theta}_{11}\right) \\
& +\left(\frac{\partial r_{0}}{\partial \theta_{22}}\right)^{2} \times \operatorname{var}\left(\hat{\theta}_{22}\right) \\
& \operatorname{var} \hat{\mathrm{r}}_{\theta}=-\frac{\theta_{12} \times \operatorname{cov}\left(\hat{\theta}_{12}, \hat{\theta}_{11}\right)}{\theta_{11}^{2} \times \theta_{22}}-\frac{\theta_{12} \times \operatorname{cov}\left(\hat{\theta}_{12}, \theta_{11}\right)}{0_{22}^{2} \times \theta_{11}}+\frac{\theta_{12}^{2} \times \operatorname{cov}\left(\theta_{11}, \theta_{12}\right)}{20_{11}^{2} \times \theta_{22}^{2}} \\
& +\frac{\operatorname{var}\left(\theta_{12}\right)}{\theta_{11} \times \theta_{22}}+\frac{\theta_{12}^{2} \times \operatorname{var}\left(\theta_{11}\right)}{4 \theta_{11}^{3} \times \theta_{22}}+\frac{\theta_{12}^{2} \times \operatorname{var}\left(\theta_{22}\right)}{4 \theta_{22}^{3} \times \theta_{11}} \text {. }
\end{aligned}
$$

On aura noté au paragraphe 14 de ce chapitre que les termes cov $\left(\theta_{12}, \hat{\theta}_{11}\right)$, $\operatorname{cov}\left(\hat{\theta}_{12}, \hat{\theta}_{22}\right) \ldots$ s'expriment en fonction des variances et covariances des termes $v_{12}, V_{q q}, v_{q q}, v_{12}(q=1,2)$. 


\section{8. - Cas de l'hypothèse de nullité de $r_{\theta}$}

C'est le cas qui répond souvent à la préoccupation du généticien : une corrélation diffère-t-elle significativement de zéro?

On est dans le cas où $\theta_{12}=0$, c'est-à-dire qu'à partir de (1) dis paragraphe 1.7, on obtient :

$$
\operatorname{var} \hat{r}_{\theta}=\frac{\operatorname{var}\left(\hat{\theta}_{12}\right)}{\theta_{11} \times \theta_{22}}=\frac{\lambda^{2} \operatorname{var}\left(V_{12}\right)+\mu^{2} \operatorname{var}\left(v_{12}\right)}{\theta_{11} \times \theta_{22}}
$$

D'après les résultats de 1.3 , nous avons :

en se souvenant que :

$$
\operatorname{var}\left(V_{12}\right)=\frac{E\left(V_{11}\right) \times E\left(V_{22}\right)+E\left(V_{12}\right)^{2}}{(p-1)}
$$

$$
E\left(V_{q q}\right)=\sigma_{e(q)}^{2}+n \sigma_{g(q)}^{2}(q=1,2)
$$

et que :

$$
E\left(V_{12}\right)=\operatorname{cov}_{e(1,2)}+n \operatorname{cov}_{g}(1,2)
$$

II vient :

$$
\operatorname{var}\left(V_{12}\right)=\frac{\left(\sigma_{\mathrm{e}(1)}^{2}+n \sigma_{\mathrm{g}(1)}^{2}\right)\left(\sigma_{\mathrm{e}(2)}^{2}+n \sigma_{\mathrm{g}(2)}\right)+\left(\operatorname{cov}_{\mathrm{e}(1,2)}+n \operatorname{cov}_{\mathrm{g}(1,2)}\right)^{2}}{(\mathrm{p}-1)}
$$

De même :

$$
\operatorname{var}\left(v_{12}\right)=\frac{\sigma_{\mathrm{e}(1)}^{2} \times \sigma_{\mathrm{e}(2)}^{2}+\left(\operatorname{cov}_{\mathrm{e}(1,2))^{2}}\right.}{\mathrm{P}(\mathrm{n}-1)}
$$

d'où l'expression de var $\widehat{\left(r_{\theta}\right)}$

$$
\begin{array}{r}
\operatorname{var}\left(\hat{r_{\theta}}\right)=\lambda^{2} \frac{\left(\sigma_{\mathrm{e}(1)}^{2}+\mathrm{n} \sigma_{\mathrm{g}(1)}^{2}\right)\left(\sigma_{\mathrm{e}(2)}^{2}+\mathrm{n} \sigma_{\mathrm{g}(2)}^{2}\right)+\left(\operatorname{cov}_{\mathrm{e}(1,2)}+\mathrm{n} \operatorname{cov}_{\mathrm{g}(1,2))^{2}}\right.}{(\mathrm{p}-1)}+ \\
+\mu^{2} \frac{\sigma_{\mathrm{e}(1)}^{2} \times \sigma_{\mathrm{e}(2)+\left(\operatorname{cov}_{\mathrm{e}(1,2))^{2}}^{2}\right.}^{\mathrm{p}(\mathrm{n}-1)}}{}
\end{array}
$$

\section{2. - Cas d'un test clonal}

Ainsi qu'il a été indiqué au paragraphe 1.4 , on se souvient que :

$$
\left.\begin{array}{l}
\sigma_{\mathrm{g}(\mathrm{q})}^{2}=\sigma_{\mathrm{G}(\mathrm{q})}^{2} \\
\sigma_{\mathrm{e}(\mathrm{q})}^{2}=\sigma_{\mathrm{E}(\mathrm{q})}^{2}
\end{array}\right\}(\mathrm{q}=1,2) \quad \begin{aligned}
& \operatorname{cov}_{\mathrm{g}(1,2)}=\operatorname{cov}_{\mathrm{G}(1,2)} \\
& \operatorname{cov}_{\mathrm{e}(1,2)}=\operatorname{cov}_{\mathrm{E}(1,2)}
\end{aligned}
$$

On a :

$$
\sigma_{\mathrm{E}(\mathrm{q})}^{2}+\sigma_{\mathrm{G}(q)}^{2}=\sigma_{\mathrm{P}(q)}^{2} \text { : variance phénotypique du caractère } q(q=1,2)
$$

$\operatorname{cov}_{E(1,2)}+\operatorname{cov}_{G(1,2)}=\operatorname{cov}_{P(1,2)}:$ covariance phénotypique des caractères 1 et 2

$$
\frac{\sigma_{G(q)}^{2}}{\sigma_{P(q)}^{2}}=h^{2} \text { est l'héritabilité au sens large du caractère } q
$$


$r_{G(1,2)}=\frac{\operatorname{cov}_{G(1,2)}}{\sqrt{\sigma_{G(1)}^{2} \times \sigma_{G(2)}^{2}}}$ : corrélation génotypique entre les caractères 1 et 2

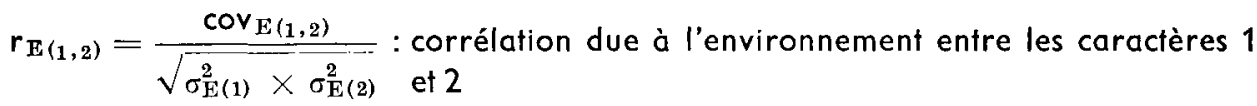

$\mathrm{r}_{\mathrm{P}(1,2)}=\frac{\operatorname{cov}_{\mathrm{P}}(1,2)}{\sqrt{\sigma_{\mathrm{P}(1)}^{2} \times \sigma_{\mathrm{P}(2)}^{2}}}:$ corrélation phénotypique entre les caractères 1 ef 2.

On s'attachera dans ce cas particulier à donner des expressions de var $\hat{r}_{G}$, var $\widehat{r}_{E}$, var $\hat{r}_{P}$. On en déterminera un seuil de signification à 5 p. 100 qui permettra de tester l'hypothèse de nullité de $r_{\theta}$ si la valeur estimée dépasse les bornes de l'intervalle ainsi défini. On fera remarquer au lecteur que nous nous sommes placés pour ce faire dans le cas de la normalité de la représentation de $\hat{r}_{\theta}$. G. H. Brown (1969) a effectué une étude par simulation de la distribution du coefficient de corrélation génétique et a conclu à la normalité approximative de sa distribution pour des héritabilités assez fortes (de l'ordre de 0,4$)$ avec un nombre élevé d'échantillons $(>1000)$, mais ses calculs s'appliquent à des estimations de $r_{G}$ par régression parents-descendants. En ce qui concerne les estimations obtenues en analyse de variance-covariance, les travaux de ce genre, qui seraient très lourds, font défaut. On ne peut faire que des parallèles avec les corrélations simples, qui deviennent assymptotiquement normales lorsque le nombre d'observations est grand, et remarquer que la détermination des seuils de $r_{G}$ faite plus loin, en supposant vérifiée la normalité, sera d'autant plus entachée d'erreur que le nombre de clones ef les héritabilités sont plus faibles.

\section{1. - Test fréquemment utilisé en France par les améliorateurs}

Afin de tester les hypothèses $r_{G}, r_{E}$ ou $r_{P}=0$, on les assimile souvent à des coefficients de corrélation dont les nombres de degrés de liberté sont les suivants :

- pour $r_{G}$ : nombre de degrés de liberté de l'effet clone : $p-1$,

- pour $r_{E}$ : nombre de degrés de liberté de l'effet individu dans clone : $p(n-1)$,

- pour $r_{P}$ : on prend, tantôt le nombre de degrés de liberté de l'erreur $(p(n-1))$, tantôt le nombre total d'individus moins un, $(p n-1)$. En vérité, comme la plupart du temps le nombre de clones est faible devant le nombre total d'individus, les tests pour $r_{P}$ sont assez voisins dans les deux éventualités.

On rejette l'hypothèse d'absence de liaison entre les deux caracières (aux niveaux génotypique, environnemental ou phénotypique) à 5 (ou 1 p. 100) si les coefficients $r_{\theta}$ estimés dépassent les valeurs seuils indiquées dans les tables classiques en fonction du nombre de degrés de liberté de $r$.

On voit qu'arec cette méthode, il n'est pas tenu compte, par exemple, des héritabilités des caractères : ainsi, rien ne nous empêche d'annoncer un $r_{G}$ non nul quand bien même l'effet clone sur l'un ou l'autre (ou les deux) caractère n'esł pas significatif. A la limite, on peut trouver, avec $\widehat{\sigma}_{\mathrm{G}(1)}^{2} \simeq 0$, un $\widehat{r}_{\mathrm{G}}$ infini ou, à tout le moins, largement supérieur à 1 en valeur absolue : $r_{\mathrm{G}}$ sera déclaré significatif.

Remarque : Nous nous sommes placés volontairement ici dans le cas d'un test clonal. Ces tests sont également ufilisés dans le cas d'études faisant intervenir des 
fratries ou demi-fratries : voir Arbez et Millier (1972), Nepveu ef al. (1978), Polge ef Illy (1968), Teissier du Cros (1976).

2.2. - Calcul de var $\left(\hat{r}_{\mathrm{G}}\right)$ sous l'hypothèse de nullité de $\mathrm{r}_{\mathrm{G}}$

Comme il a été indiqué au paragraphe 1.5 , nous avons :

$$
\lambda=\frac{1}{n}, \quad \mu=-\frac{1}{n} .
$$

En outre $\theta_{11}$ et $\theta_{22}$ représentent ici respectivement $\sigma_{G(1)}^{2}$ et $\sigma_{G(2)}^{2}$.

En se souvenant que $\operatorname{cov}_{G}=0$ sous l'hypothèse de nullité de $r_{G}$, l'expression (2) de 1.8 s'écrif :

$$
\begin{aligned}
& \operatorname{var}\left(\hat{r}_{G}\right)=\frac{1}{n^{2}}\left(\frac{\left(\sigma_{E(1)}^{2}+n \sigma_{G(1)}^{2}\right)\left(\sigma_{E(2)}^{2}+n \sigma_{G(2)}^{2}\right)+\left(\operatorname{cov}_{E(1,2)}\right)^{2}}{(P-1) \times \sigma_{G(1)}^{2} \times \sigma_{G(2)}^{2}}+\right. \\
& \left.+\frac{\sigma_{\mathrm{E}(1)}^{2} \times \sigma_{\mathrm{E}(2)}^{2}+\left(\operatorname{cov}_{\mathrm{E}(1,2)^{2}}\right.}{\mathrm{P}(\mathrm{n}-1) \times \sigma_{\mathrm{G}(1)}^{2} \times \sigma_{\mathrm{G}(2)}^{2}}\right) \\
& \operatorname{var}\left(\hat{r}_{G}\right)=\frac{1}{n^{2}}\left[\frac{1}{(P-1)}\left(\left(\frac{1}{h_{1}^{2}}+(n-1)\right)\left(\frac{1}{h_{2}^{2}}+(n-1)\right)+r_{E}^{2}\left(\frac{1-h_{1}^{2}}{h_{1}^{2}}\right)\left(\frac{1-h_{2}^{2}}{h_{2}^{2}}\right)\right)+\right. \\
& \left.+\frac{1}{P(n-1)}\left(\left(\frac{1-h_{1}^{2}}{h_{1}^{2}}\right)\left(\frac{1-h_{2}^{2}}{h_{2}^{2}}\right)+r_{E}^{2}\left(\frac{1-h_{1}^{2}}{h_{1}^{2}}\right)\left(\frac{1-h_{2}^{2}}{h_{2}^{2}}\right)\right)\right]
\end{aligned}
$$

En prenant

$$
A=\sqrt{\frac{1-h_{1}^{2}}{h_{1}^{2}}} \text { et } B=\sqrt{\frac{1-h_{2}^{2}}{h_{2}^{2}}}
$$

afin d'alléger la formule, il vient :

$$
\operatorname{var}\left(r_{G}\right)=\frac{1}{n^{2}}\left[\frac{\left(A^{2}+n\right)\left(B^{2}+n\right)+r_{E}^{2} A^{2} B^{2}}{(P-1)}+\frac{A^{2} B^{2}\left(1+r_{E}^{2}\right)}{P(n-1)}\right]
$$

En se plaçant dans le cas de la normalité de la représentation de $\widehat{r}_{\mathrm{G}}$, on peut en déterminer un seuil de signification à 5 p. 100 qui est en valeur absolve :

$$
\frac{1,96}{n} \sqrt{\frac{\left(A^{2}+n\right)\left(B^{2}+n\right)+r_{E}^{2} A^{2} B^{2}}{(p-1)}+\frac{A^{2} B^{2}\left(1+r_{E}^{2}\right)}{P(n-1)}} .
$$

On conclura à la non-nullité de $r_{G}$ si la valeur estimée dépasse les bornes de l'intervalle ainsi défini.

On voit que le seuil de signification dépend certes du nombre de clones ( $P$ ), mais également des héritabilités des deux caractères, du coefficient de corrélation due à l'environnement et du nombre d'individus par clone. On remarque que l'approximation consistant à ne tenir compte que de $p$ pour déterminer un seuil de signification revient à faire dans l'expression (1) :

$$
r_{\mathrm{E}}=0 \quad \text { et } \quad A=B=0 \quad\left(\text { soit } h_{1}^{2}=h_{2}^{2}=1\right) \text {. }
$$




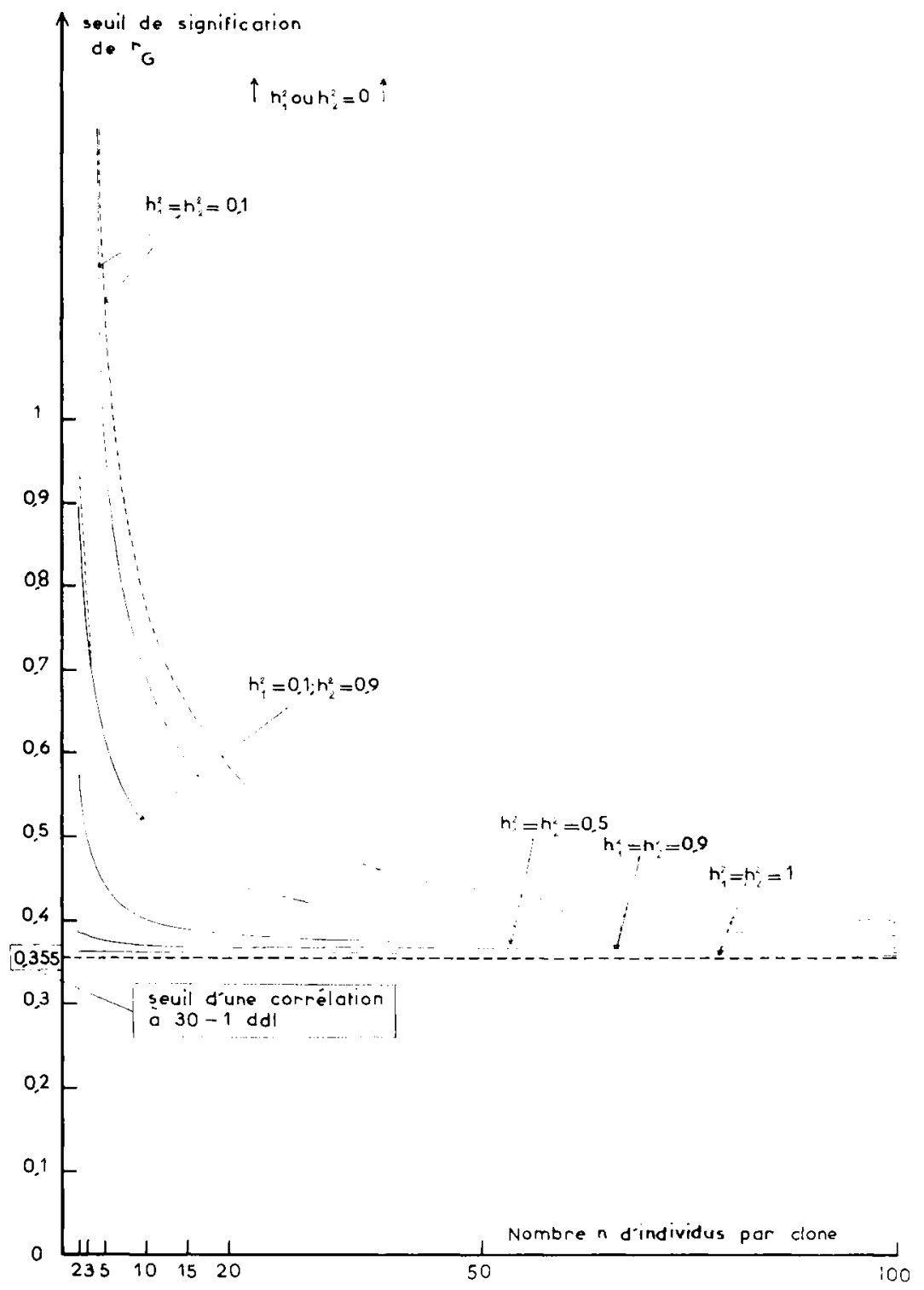

FIG. 1. - Seuil de signification contre zéro à 5 p. 100 du coefficient de corrélation génolypique $r_{G}$ en fonction des héritabilités au sens large des caractères, du coefficient de corrélation due au milieu $\mathrm{r}_{\mathrm{E}}$ et du nombre $n$ d'individus par clone.

p, nombre de clones, égal à $30 ;-r_{\mathrm{E}}=0 ; \cdots, \mathrm{r}_{\mathrm{E}} \mid=1$.

Significance level against zero (at 5 p. 100 level) of genotypic correlation coefficient $r_{G}$ in terms of brood sense heritabilities of two characters, environmental correlation coefficient $\mathrm{r}_{\mathrm{E}}$ and size $\mathrm{n}$ of each clone. $\mathrm{p}$, number of clones equal to $30 ;-r_{\mathrm{E}}=0 ; \ldots-\left|\mathrm{r}_{\mathrm{E}}\right|=1$. 


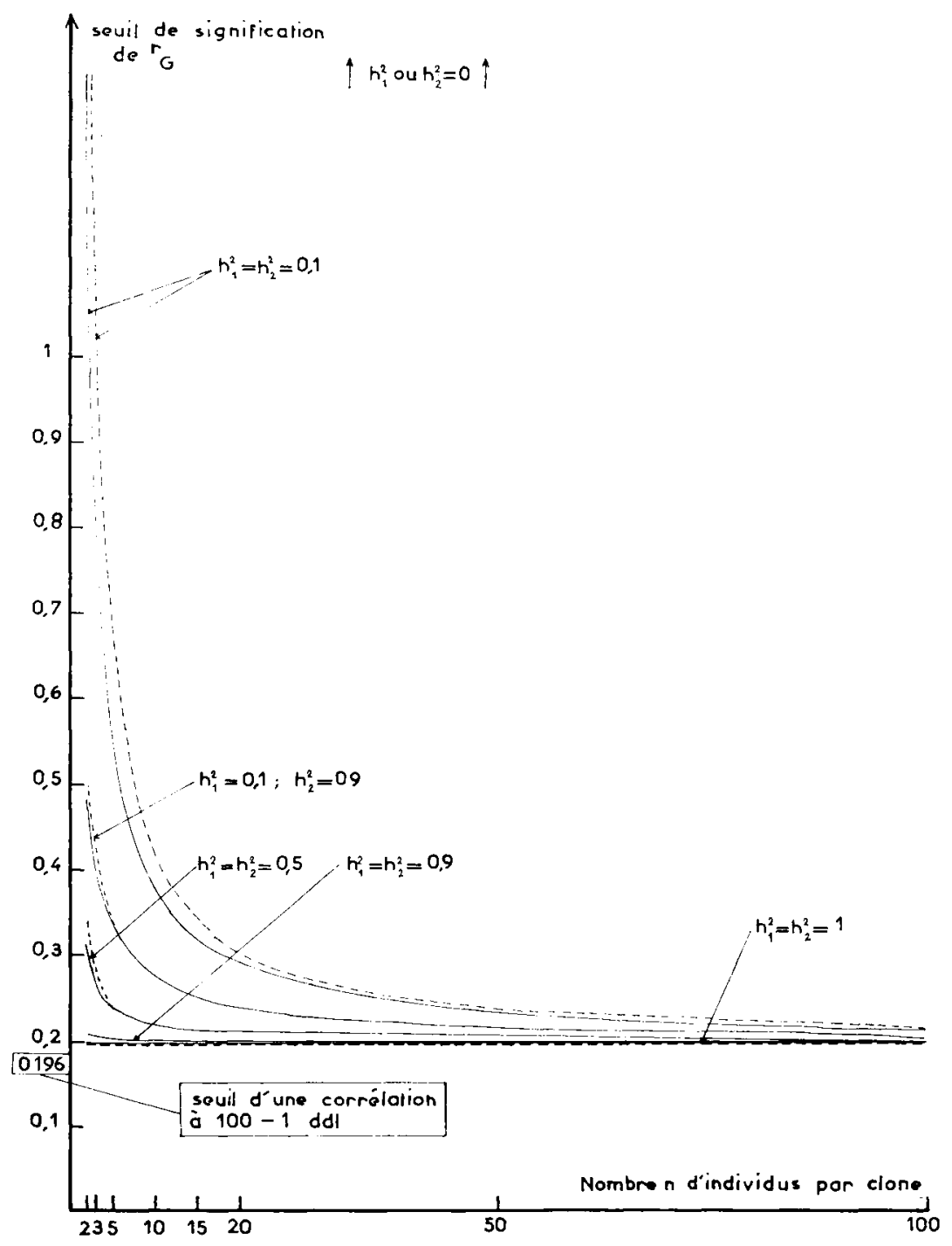

FIG. 2. - Seuil de signification contre zéro d̀ 5 p. 100 du coefficient de corrélation génotypique $\mathbf{r}_{\mathrm{G}}$ en fonction des héritabilités ou sens large des caractères, du coefficient de corrélation due au milieu $r_{\mathrm{E}}$ ef du nombre $n$ d'individus par clone.

$\mathrm{p}$, nombre de clones, égal à $100 ;-r_{E}=0 ; \ldots .-r_{E} \mid=1$.

Significance level against zero (at 5 p. 100 level) of genotypic correlation coefficient $r_{G}$ in ferms of broad sense heritabilities of two characters, environmental correlation coefficient $r_{E}$ and size $n$ of each clone. P, number of clones equal to $100 ;-r_{E}=0 ;-\cdots\left|r_{E}\right|=1$. 
En effet (1) s'écrit $\frac{1,96}{\sqrt{\mathrm{P}-1}}$, valeur voisine du seuil de signification d'un coefficient de corrélation à $p-1$ degrés de liberté lorsque $p>30$. En d'autres termes, l'approximation n'est licite que lorsque seuls des effets génétiques sont responsables de la variabilité des deux caractères. Il ne sert alors à rien de multiplier le nombre d'individus par clone pour estimer $r_{G}$ puisque, les héritabilités étant égales à 1, tous les individus d'un même clone sont rigoureusement identiques.

Les figures 1 et 2 comparent, respectivement pour $p=30$ et $p=100$ les seuils utilisés en prenant pour $r_{G}$ la signification contre zéro d'un coefficient de corrélation à $p-1$ degrés de liberté et les valeurs-seuils telles que nous les avons définies plus haut.

Le trait horizontal correspond au seuil de signification à $5 p$. 100 avec $p-1$ degrés de liberté. Les courbes en traits pleins correspondent à $r_{\mathrm{E}}=0$, celles en traits pointillés à $\left|r_{E}\right|=1$.

Nous voyons que l'approximation faite ordinairement est particulièrement dangereuse lorsque :

- les héritabilités sont faibles,

- le nombre d'individus par clone est faible.

Les valeurs des seuils sont peu sensibles aux variations de $r_{\mathrm{E}}$.

Exemple : Prenons l'exemple de l'estimation d'une corrélation génotypique avec 100 clones (avec $r_{E}=0$ pour fixer les idées). La méthode approximative va rejeter l'hypothèse de nullité de $r_{G}$ à 5 p. 100 , quels que soient $n, h_{1}^{2}$ et $h_{2}^{2}\left(\right.$ et $\left.r_{E}\right)$ si $r_{G}$ dépasse 0,195 .

If faut au contraire ne déclarer $r_{G}$ significativement différent de zéro que s'il dépasse les valeurs suivantes:

TABLEAU 4

Seuil de signification contre zéro au niveau 5 p. 100 de $r_{G}$ ovec $p=100$ ef $r_{E}=0$

Signifiance level against zero (at 5 p. 100 level) of $\mathrm{r}_{\mathrm{G}}$ with $\mathrm{P}=100$ and $\mathrm{r}_{\mathrm{IE}}=0$

\begin{tabular}{|c|c|c|c|c|c|}
\hline \multirow{2}{*}{ Valeurs des héritabilités } & \multicolumn{5}{|c|}{ Nombre d'individus par clone } \\
\hline & 2 & 5 & 10 & 20 & 50 \\
\hline$h_{1}^{2}=h_{2}^{2}=-0,1 \ldots \ldots \ldots \ldots \ldots \ldots$ & 1,398 & 0,579 & 0,379 & 0,286 & 0,232 \\
\hline$h_{1}^{2}=0,1 ; h_{2}^{2}=0,5 \ldots \ldots \ldots \ldots \ldots$ & 0,638 & 0,366 & 0,285 & 0,243 & 0,217 \\
\hline$h_{1}^{2}=h_{2}^{2}==0,5 \quad \ldots \ldots \ldots \ldots \ldots \ldots$ & 0,312 & 0,237 & 0,217 & 0,207 & 0,201 \\
\hline$h_{1}^{2} \quad 0,1 ; h_{2}^{2}=0,9 \ldots \ldots \ldots \ldots$ & 0,485 & 0,334 & 0,273 & 0,238 & 0,215 \\
\hline$h_{1}^{2}=0,5 ; h_{2}^{2}=0,9 \ldots \ldots \ldots \ldots \ldots$ & 0,250 & 0,219 & 0,208 & 0,203 & 0,199 \\
\hline$h_{1}^{2}=h_{2}^{2}=0,9 \ldots \ldots \ldots \ldots \ldots \ldots$ & 0,209 & 0,202 & 0,199 & 0,198 & 0,198 \\
\hline
\end{tabular}

En prenant 30 clones (et toujours $r_{E}=0$ ), on aura les seuils suivants (alors que la méthode approximative donne une valeur limite de 0,355 ) : 


\section{TABLEAU 5}

Seuil de signification contre zéro au niveau 5 p. 100 de $r_{6}$ avec $p-30$ et $r_{\mathrm{E}}=0$

Significance level against zero (at 5 p. 100 level) of $r_{G}$ with $P=30$ and $r_{E}=0$

\begin{tabular}{|c|c|c|c|c|c|}
\hline \multirow{2}{*}{ Valeurs des héritabilités } & \multicolumn{5}{|c|}{ Nombre d'individus par clone } \\
\hline & 2 & 5 & 10 & 20 & 50 \\
\hline$h_{1}^{2}=h_{2}^{2}=0,1 \ldots \ldots$. & 2,569 & 1,069 & 0,700 & 0,529 & 0,429 \\
\hline$h_{1}^{2}=0,1 ; h_{2}^{2}=0,5 \quad \ldots$ & 1,175 & 0,676 & 0,527 & 0,449 & 0.400 \\
\hline$h_{1}^{2}=h_{2}^{2}=0,5 \ldots \ldots \ldots$ & 0,574 & 0,438 & 0,401 & 0,382 & 0,371 \\
\hline$h_{1}^{2}=0,1 ; h_{2}^{2}=0,9 \quad \ldots$ & 0,895 & 0,616 & 0,505 & 0,440 & 0,396 \\
\hline $\mathrm{h}_{1}^{2}=0,5 ; \mathrm{h}_{2}^{2}=0,9 \quad \ldots \ldots \ldots$. & 0,462 & 0,404 & 0,384 & 0,374 & 0,368 \\
\hline$h_{1}^{2}=0,9 ; h_{2}^{2}=0.9 \ldots \ldots \cdot$ & 0,385 & 0,372 & 0,368 & 0,367 & 0,365 \\
\hline
\end{tabular}

2.3. - Cas de $r_{E}$ sous l'hypothèse nulle

$\mathbf{r}_{\mathrm{E}}$ étant estimé par la corrélation intraclone obtenue dans l'analyse de variancecovariance, on devrait trouver une variance d'échantillonnage égale à celle d'un coefficient de corrélation à $p(n-1)$ degrés de liberté qui est le nombre de degrés de liberté de la variance intraclone.

Dans la formule (2) du paragraphe $1.8, \theta_{11}$ et $\theta_{22}$ représentent cette fois $\sigma_{\mathrm{E}(1)}^{2}$ ef $\sigma_{\mathrm{E}}^{2}(2)$.

D'après le paragraphe 1.5 , on $a \lambda=0$ ef $\mu=1$.

Il vient :

$$
\operatorname{var}\left(\hat{\left.r_{\mathrm{E}}\right)}=\begin{array}{l}
\sigma_{\mathrm{E}(1)}^{2} \times \sigma_{\mathrm{E}(2)}^{2}+\operatorname{cov}_{\mathrm{E}(1,2)} \\
P(\mathrm{n}-1) \times \sigma_{\mathrm{E}(1)}^{2} \times \sigma_{\mathrm{E}(2)}^{2}
\end{array} .\right.
$$

Sous l'hypothèse de nullité de $r_{\mathrm{E}}$, on $a \operatorname{cov}_{\mathrm{E}(1,2)}=0$, d'où

$$
\operatorname{var}\left(\hat{r}_{E}\right)=\frac{1}{\mathrm{P}(\mathrm{n}-1)} \text {. }
$$

On reconnaît bien là la variance d'échantillonnage d'un coefficient de corrélation à $\mathrm{p}(\mathrm{n}-1)$ degrés de liberté sous l'hypothèse de nullité.

2.4. - Cas de $r_{P}$ sous l'hypothèse nulle

Cette fois nous avons

$$
\begin{aligned}
\lambda & =\frac{1}{\mathrm{n}} \\
\mu & =\frac{\mathrm{n}-1}{\mathrm{n}} \\
0_{11} & =\sigma_{\mathrm{P}(1)}^{2} \\
\theta_{22} & =\sigma_{\mathrm{P}(2)}^{2} \\
\operatorname{cov}_{\mathbf{P}(1,2)}=0 & \text { (hypothèse de nullité de } r_{\mathrm{P}} \text { ). }
\end{aligned}
$$


En reprenant (2) de 1.8, il vient :

$$
\begin{array}{r}
\operatorname{var}\left(\hat{r}_{\mathrm{P}}\right)=\frac{1}{n^{2}}\left[\frac{\left(\sigma_{\mathrm{E}(1)}^{2}+n \sigma_{\mathrm{G}(1)}^{2}\right)\left(\sigma_{\mathrm{E}(2)}^{2}+n \sigma_{\mathrm{G}(2)}^{2}\right)+\left(\operatorname{cov}_{\mathrm{E}(1,2)}+n \operatorname{cov}_{\mathrm{G}(1,2)}\right)^{2}}{(\mathrm{P}-1) \times \sigma_{\mathrm{P}(1)}^{2} \times \sigma_{\mathrm{P}(2)}^{2}}+\right. \\
\left.+\frac{(n-1)}{P} \times \frac{\sigma_{\mathrm{E}(1)}^{2} \times \sigma_{\mathrm{E}(2)}^{2}+\left(\operatorname{cov}_{\mathrm{E}(1,2)}\right)^{2}}{\sigma_{\mathrm{P}(1)}^{2} \times \sigma_{\mathrm{P}(2)}^{2}}\right]
\end{array}
$$

$\operatorname{var}\left(\hat{r}_{P}\right)=\frac{1}{n^{2}}\left[\frac{\left(1+(n-1) h_{1}^{2}\right)\left(1+(n-1) h_{2}^{2}\right)+(n-1)^{2} r_{G}^{2} h_{1}^{2} h_{2}^{2}}{(P-1)}+\right.$

$$
\left.+\frac{(n-1)}{P} \times\left(\left(1-h_{1}^{2}\right)\left(1-h_{2}^{2}\right)+r_{G}^{2} h_{1}^{2} h_{2}^{2}\right)\right] .
$$

En admettant la normalité de la représentation de $\widehat{r}_{P}$, le sevil de signification à 5 p. 100 de $r_{P}$ vaut :

$$
\begin{aligned}
& \frac{1,96}{n} \sqrt{\frac{\left(1+(n-1) h_{1}^{2}\right)\left(1+(n-1) h_{2}^{2}\right)+(n-1)^{2}}{(p-1)} r_{G}^{2} h_{1}^{2} h_{2}^{2}}+\cdots \\
& \cdots \frac{n-1}{p} \times\left(\left(1-h_{1}^{2}\right)\left(1-h_{2}^{2}\right)+r_{G}^{2} h_{1}^{2} h_{2}^{2}\right) .
\end{aligned}
$$

On pourra rejeter l'hypothèse de nullité de $r_{\mathrm{P}}$ si la valeur estimée dépasse les bornes de l'intervalle ainsi défini.

Les figures 3 et 4 comparent pour quelques valeurs de $h_{1}^{2}$, $h_{2}^{2}$ ef $r_{G}$ les seuils obtenus en prenant pour $r_{P}$ la signification contre zéro d'un coefficient de corrélation à $n p-1$ degrés de liberté et les valeurs-seuils telles que nous les avons définies plus haut.

- La figure 3 correspond à une population totale de deux cents individus. Le trait horizontal représente le seuil de signification à 5 p. 100 d'un coefficient de corrélation à 199 degrés de liberté $(0,139)$; les courbes en traits pleins correspondent à $r_{G}=0$; celles en pointillés à $\left|r_{G}\right|=1$.

- La figure 4 correspond à une population de mille individus. Le trait horizontal représente le seuil de signification à 5 p. 100 d'un coefficient de corrélation à 999 degrés de liberté $(0,062)$. Les courbes en traits pleins et en pointillés correspondent respectivement à $r_{G}=0$ et $\left|r_{G}\right|=1$.

Au nombre total d'individus donné, on voit que la méthode qui consiste à prendre $n p-1$ degrés de liberté pour $r_{P}$ nous conduit à déclarer $n_{P}$ non nul de façon erronée ; le risque encouru est d'autant plus important que $r_{G}$ et les héritabilités sont plus grandes, surtout lorsqu'on prend beaucoup d'individus par clone. Ceci est assez normal : lorsque le contrôle génétique est fort, c'est la variabilité clonale qui représente l'essentiel de la variance phénotypique. On a donc intérêt à avoir un grand nombre de clones pour estimer $\mathbf{r}_{\mathrm{p}}$.

\section{3. - Un exemple}

Nepveu, Keller et Tessier du Cros (1978) ont étudié vingt-huit clones de Populus euramericana pour quelques caractéristiques de vigueur et de qualité du bois. 


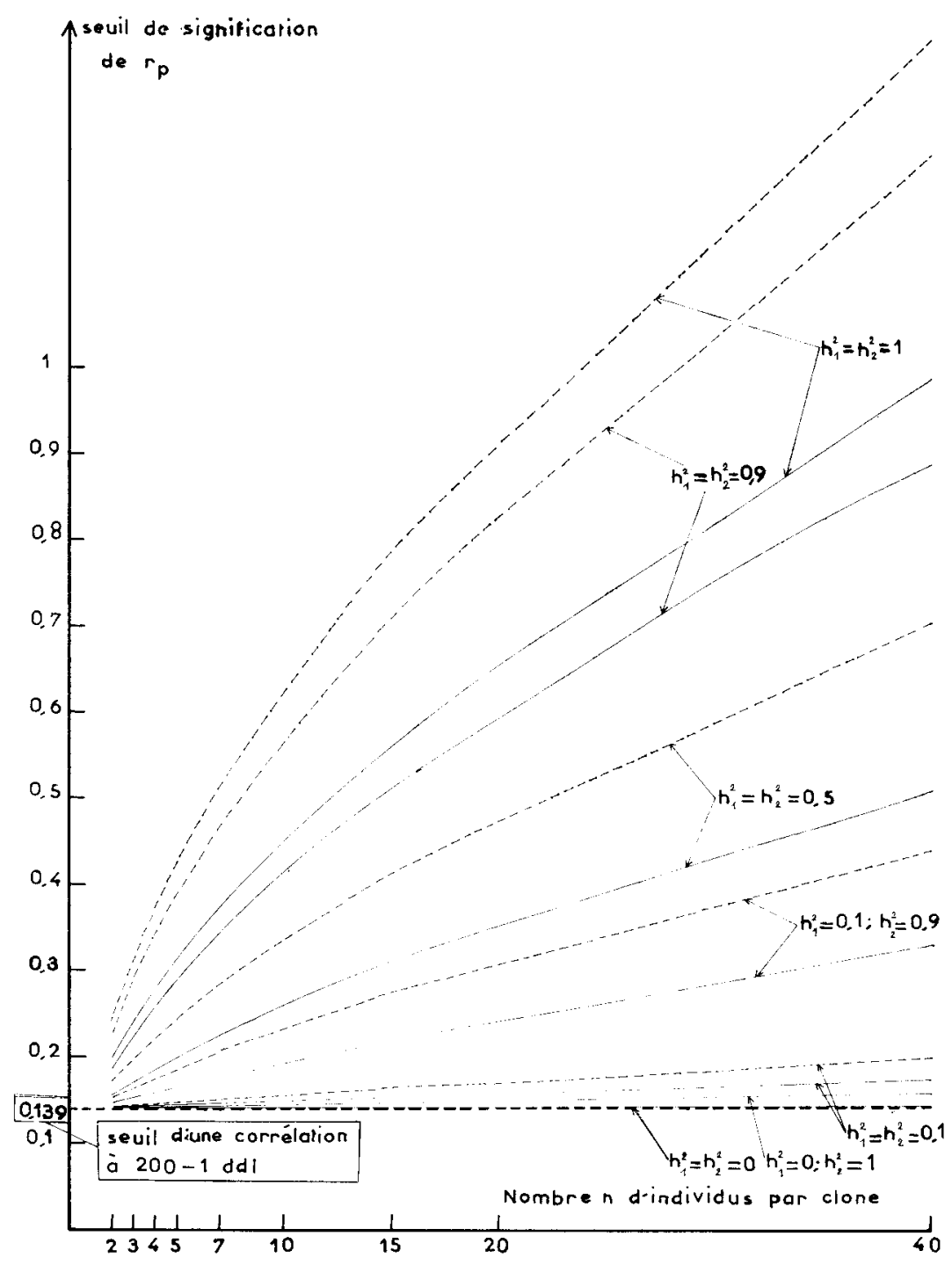

FIG. 3. - Seuil de signification contre zéro à 5 p. 100 du coefficient de corrélation phénotypique $r_{P}$ en fonction des héritabilités au sens large des caractères, du coefficient de corrélation génotypique $r_{\mathrm{G}}$ ef du nombre $\mathbf{n}$ d'individus par clone.

np, nombre total d'individus fixé, égal à $200 ;-r_{G}=0 ;-\ldots\left|r_{G}\right|=1$.

Significance level against zero (at 5 p. 100 level) of phenofypic correlation coefficient $r_{P}$ in terms of broad sense heritabilities of two characters, genotypic correlation coefficient $r_{G}$ and size $n$ of each clone.

$n p$, total number of individuals equal to $200 ;-r_{G}=0 ; \cdots-r_{G} \mid=1$. 


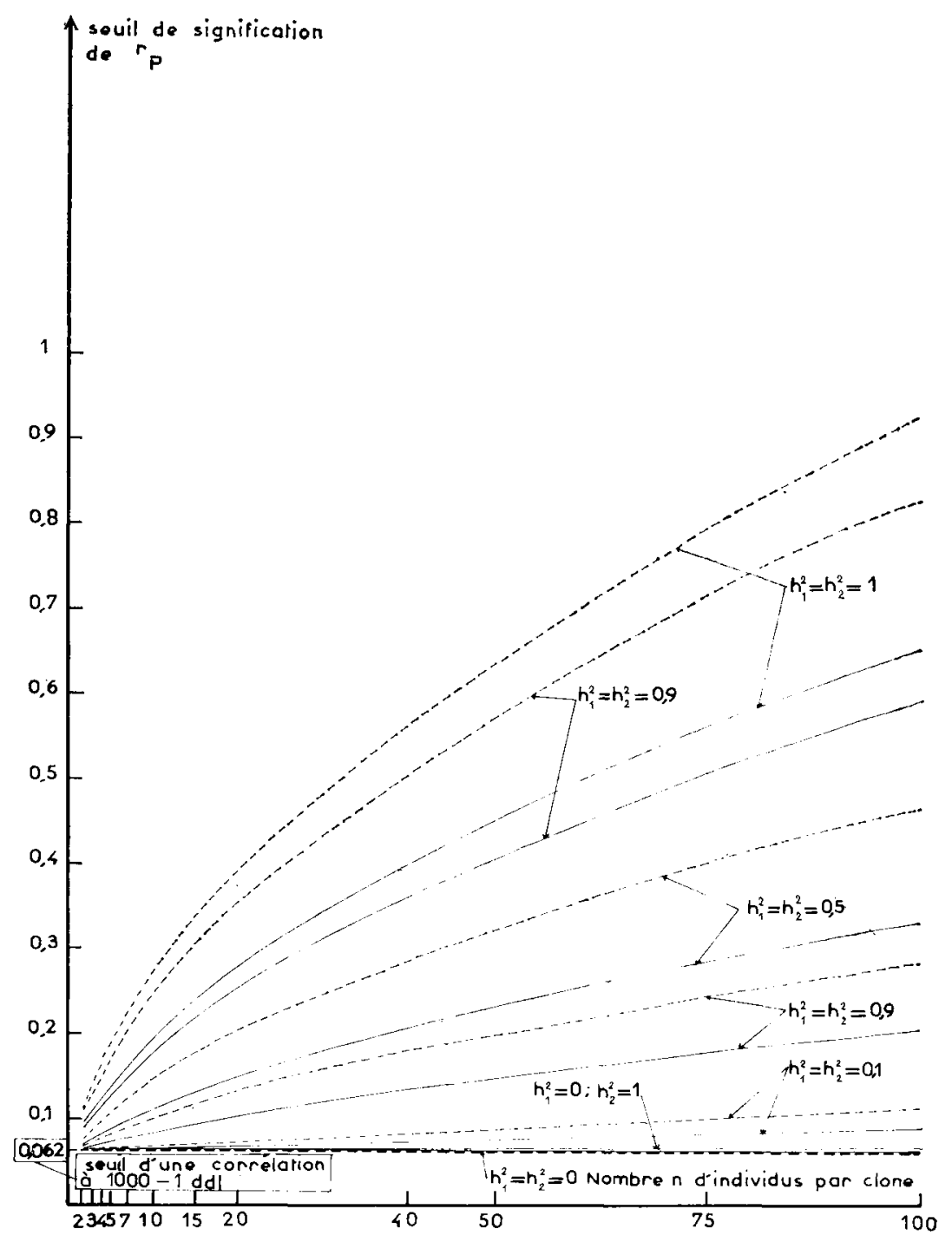

FIG. 4. - Seuil de signification contre zéro à 5 p. 100 du coefficient de corrélation phénotypique $r_{p}$ en fonction des héritabilités au sens large des coractères, du coefficient de corrélation génotypique $r_{0}$ et du nombre $\mathbf{n}$ d'individus par clone.

$\mathrm{np}$, nombre total d'individus fixé, égal à $1000 ;-\mathrm{r}_{\mathrm{G}}=0 ; \ldots-\mathrm{r}_{\mathrm{G}} \mid=1$.

Significance level against zero (at 5 p. 100 level) of phenotypic correlation caefficient $r_{p}$ in terms of broad sense heritabilities of two characters, genotypic correlation coefficient $r_{\mathrm{G}}$ and size $\mathrm{n}$ of each clone.

$n p$, total number of individuals equal to $1000 ;-r_{G}=0 ; \ldots . .\left|r_{G}\right|=1$. 
Le nombre d'individus par clone était égal à 2 . Les estimations des héritabilités au sens large sont les suivantes :

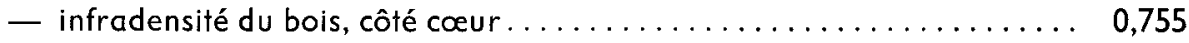

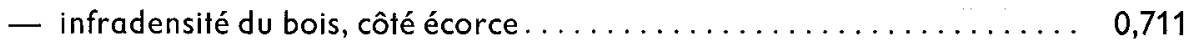

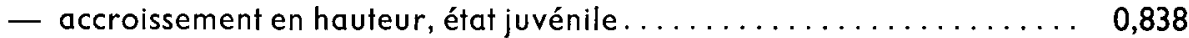

— accroissement en circonférence, état juvénile . . . . . . . . . . . . 0,824

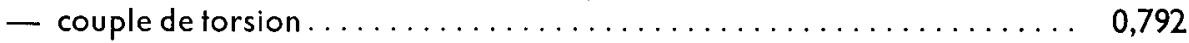

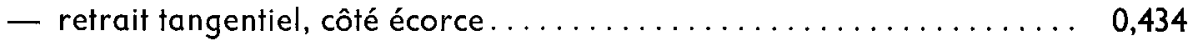

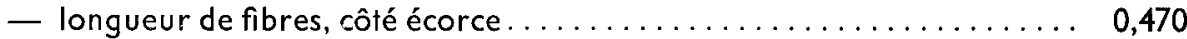

- accroissement en hauteur, état adulte . . . . . . . . . . . . . . . 0,801

— accroissement en circonférence, état adulte . . . . . . . . . . . . . 0,901

Par la méthode approximative, $r_{G}, r_{E}$ et $r_{P}$ sont déclarées non nuls à 5 p. 100 s'ils dépassent les valeurs-seuils $0,367\left(r_{G}\right), 0,361\left(r_{E}\right)$ et $0,261\left(r_{P}\right)$ qui correspondent respectivement à un nombre de degrés de liberté de $p-1=27, p(n-1)=28$, $\mathrm{pn}-1=55$.

En réalité, les valeurs-seuils obtenues pour $r_{G}$ et $r_{P}$, en remplaçant $h_{1}, h_{2}, r_{E}$ et $r_{G}$ par leurs estimations, sont, suivant les couples de caractères considérés, les suivantes :

TABLEAU 6

Seuils de signification de $r_{G}$ et $r_{P}$ dans quelques cas

Significance level for $\mathbf{r}_{\mathrm{G}}$ and $\mathbf{r}_{\mathrm{P}}$ in some cases

\begin{tabular}{|c|c|c|}
\hline & $\begin{array}{c}\text { Valeur-seuil } \\
r_{G}\end{array}$ & $\begin{array}{c}\text { Valeur-seuil } \\
r_{\mathrm{P}}\end{array}$ \\
\hline 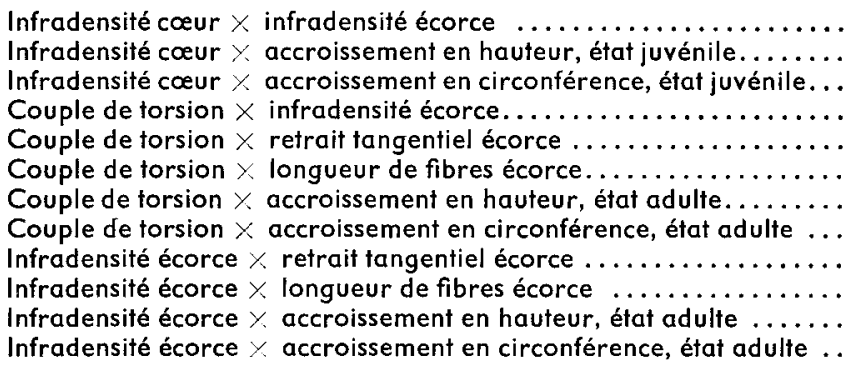 & $\begin{array}{l}0,452 \\
0,428 \\
0,431 \\
0,445 \\
0,530 \\
0,512 \\
0,428 \\
0,414 \\
0,551 \\
0,533 \\
0,426 \\
0,426\end{array}$ & $\begin{array}{l}0,355 \\
0,351 \\
0,341 \\
0,360 \\
0,320 \\
0,322 \\
0,342 \\
0,351 \\
0,315 \\
0,325 \\
0,334 \\
0,344\end{array}$ \\
\hline
\end{tabular}

Remarque : Pour calculer var $\hat{r}_{G}$ et var $\hat{r}_{P}$, on a remplacé $h_{1}^{2}, h_{2}^{2}, r_{E}$ et $r_{P}$ par leurs estimations fournies dans les analyses de variance-covariance des données observées. On doit normalement utiliser les vraies valeurs de ces coefficients que l'on ignore la plupart du temps. On sait en revanche construire un intervalle de confiance exact de $h_{1}^{2}, h_{2}^{2}$ (Bogyo et Becker, 1963) et de $r_{E}$ (Lamotte, 1962).

On est alors capable, la variance d'échantillonnage de $r_{G}$ variant de façon simple avec $h_{1}^{2}, h_{2}^{2}$ et $r_{E}$, de déterminer une valeur minima et une valeur maxima du seuil de signification de $r_{G}$ en remplaçant, dans la formule donnant (var $\hat{r}_{G}$ ), les paramètres $h_{1}^{2}, h_{2}^{2}$ et $r_{E}$ par les bornes de leurs intervalles de confiance qui minimisent ou maximisent la valeur-seuil de $r_{G}$. 
Les figures 1 et 2 montrent qu'une borne supérieure du seuil de signification de $r_{G}$ est obtenue avec les bornes inférieures de $h_{1}^{2}$ et $h_{2}^{2}$ ainsi qu'avec la borne supérieure - en valeur absolue - de $r_{\mathrm{E}}$. La borne inférieure du seuil est obtenue avec les bornes supérieures de $h_{1}^{2}$ et $h_{2}^{2}$ et avec la borne inférieure en valeur absolve de $r_{E}$.

On fera cependant deux remarques pour mettre en garde le lecteur :

- le test clonal est particulièrement simple puisque $r_{E}$, estimé par la corrélation intraclone, a un intervalle de confiance bien connu (ce n'est pas le cas de $r_{G}$ et $r_{P}$ ). Dans le cas de fratries ou semi-fratries, on ne connaît pas d'intervalle de confiance exact de $r_{E}$. L'extrapolation à d'autres cas que le test clonal n'est donc pas correcte.

- on a défini un intervalle de confiance très large pour le seuil de signification de $r_{G}$. En effet, si les infervalles de $h_{1}^{2}, h_{2}^{2}$ et $r_{E}$ sont bien définis à $5 p$. 100 (pour fixer les idées), les liaisons entre $h_{1}^{2}, h_{2}^{2}$ et $r_{E}$ font que la probabilité :

(bornes inférieures de $\left.h_{1}^{2}, h_{2}^{2}, r_{E}\right)<\left(h_{1}^{2}, h_{2}^{2}, r_{E}\right)<$ (bornes supérieures de $h_{1}^{2}, h_{2}^{2}, r_{E}$ ) est supérieure à 0,95 .

A titre d'exemple, et pour fixer les idées, prenons le cas de la liaison infradensité du bois, côté cœeur-infradensité du bois, côté écorce. On avait :

$$
r_{\mathrm{E}}=-0,170, h_{1}^{2}=0,755, h_{2}^{2}=0,711 .
$$

L'intervalle de confiance de $r_{\mathrm{E}}$ à 5 p. 100 est d'après Lamotte (1962, op. cit.) :

$$
-0,445<r_{E}<+0,295 \text {. }
$$

Les intervalles de confiance de $h_{1}^{2}$ et $h_{2}^{2}$ sont d'après Bogyo ef Becker (1963, op. cit.) :

$$
0,540<h_{1}^{2}<0,877, \quad 0,469<h_{2}^{2}<0,853 .
$$

L'expression de la limite supérieure du seuil de signification de $r_{G}$ est obtenue avec $r_{E}=0,445 ; h_{1}^{2}=0,540 ; h_{2}^{2}=0,469$. Elle vaut 0,602 .

La limite inférieure est donnée en faisant $r_{E}=0 ; h_{1}^{2}=0,877$ et $h_{2}^{2}=0,853$. Elle est égale à 0,408 .

Ces deux valeurs : 0,408 et 0,602 encadrent bien la valeur 0,452 du seuil de signification précédemment trouvé en remplaçant $h_{1}^{2}, h_{2}^{2}$ et $r_{E}$ par leurs estimations.

On peut donc dire que le seuil de signification de $r_{G}$ est compris entre 0,408 et 0,602 (avec une probabilité supérieure à 0,95 ). Cet intervalle n'encadre pas, on le remarquera, la valeur-seuil couramment utilisée en France qui vaut ici 0,367 (seuil de signification contre 0 à 5 p. 100 d'un coefficient de corrélation à $p-1=27$ degrés de liberté).

\section{4. - Conclusion}

Dans le cas d'un test clonal, la détermination des seuils de signification des coefficients de corrélation génotypique et phénotypique à l'aide des seules caractéristiques du dispositif (nombre de clones, nombre d'individus par clone) conduit toujours à rejeter abusivement l'hypothèse de nullité de $r_{G}$ ou $r_{P}$.

- Pour la corrélation génotypique, l'erreur commise croît, pour un nombre de clones donné, à mesure que les héritabilités et le nombre d'individus par clone dimi- 
nuent. Lorsque tous ces paramètres sont fixés, l'influence de $r_{E}$, corrélation due à l'environnement, est faible.

- Pour la corrélation phénotypique, en se plaçant à nombre total d'individus fixé, l'erreur reste faible pour le cas d'un contrôle génotypique minime quel que soit le nombre d'individus par clone. En revanche, elle est importante lorsque les héritabilités sont fortes et le nombre de clones faible. Lorsque tous ces paramètres sont fixés, l'erreur croît assez sensiblement avec $\left|\mathbf{r}_{\mathbf{G}}\right|$.

Ces considérations pourraient être étendues, à l'aide des formules générales rappelées dans l'article, aux cas de tests faisant intervenir des fratries ou semi-fratries.

On rappellera au lecteur les approximations faites, notamment en ce qui concerne le caractère normal des représentations de $\hat{r}_{G}$ et $\hat{r}_{P}$.

Le problème des intervalles de confiance de paramètres génétiques mériterait d'autres développements, en particulier en ce qui concerne les estimations fondées sur l'analyse de plans de croisement de modèles variés.

Reçu pour publication en avril 1979.

\section{Summary}

Significance level for the genotypic, phenotypic and environmental correlation coefficients. Clonal test assumption

This paper gives a very simple application of research developed by Tallis and Scheinberg.

If recalls the expression of the sampling variance of correlation coefficient estimation (genetic, genotypic, environmental and phenotypic) calculated from analyses of variance and covariance.

It mentions in detail the case of a clonal test and shows that a serious error may occur when the significance level of the genotypic and phenotypic correlations is appreciated by means of clonal test characteristics only : number of clones, size of each clone.

\section{Références bibliographiques}

ARBEZ M., MILLIER C., 1972. Variabilité, héritabilité ef corrélations entre caractères chez de jeunes pins laricio de Calabre (Pinus nigra Arn, ssp. laricio, var Calabrica). Conséquences et problèmes des indices de sélection. IUFRO Genetics, SABRAO joint Symposia, Tokyo.

BOGYO T. P., BECKERW. A., 1963. Exact confidence intervals for genetic heritability estimated from paternal half-sib correlations. Biometrics, 19, 494-496.

BROWN G. H., 1969. An empirical study of the distribution of the sample genetic correlation coefficient. Biometrics, 25, n $^{\circ} 1$.

FISHER R. A. 1928. Moments and products moments of sampling distributions. Proc. Lond. math. Soc., 30, 199-238.

KENDALL M. G., STUART A., 1963. The advanced theory of statistics. Vol. 1, 2nd Ed. Griffin, London, Hafner publishing Company, New York.

LAMOTTE M., 1962. Initiation aux méthodes statistiques en biologie, Masson et Cie, $2^{\mathrm{e}}$ édition.

NEPVEU G., KELLER R., TEISSIER du CROS E., 1978. Sélection juvénile pour la qualité du bois chez certains peupliers noirs. Ann. Sci. forest., 35 (1), 69-92.

POLGE H., ILLY G., 1968. Héritabilité de la densité du bois et corrélations avec la croissance étudiées à l'aide de tests non destructifs sur plants de Pins maritimes de quatre ans. Silvae Genetica, 17, 5-6, 173-181.

SCHEINBERG E., 1966. The sampling variance of the correlation coefficients estimated in genetic experiments. Biometrics, 22, 187-191.

TALLIS G. M., 1959. Sampling errors of genetic correlation coefficients calculated from analyses of variance and covariance. Aust. Jour. of Stat. 1, 35-43.

TEISSIER du CROS E., 1976. Premiers enseignements d'un test de descendances de Picea Abies Karts des Vosges (peuplement du Kertoff). Ann. Sci. forest., 32 (3), 143-155. 